\title{
Non-invasive brain stimulation: the next frontier in psychiatry
}

\author{
Sebastian Walther ${ }^{1} \cdot$ Chris Baeken ${ }^{2,3,4}$
}

Published online: 12 January 2021

(c) Springer-Verlag GmbH, DE part of Springer Nature 2021

Psychiatry has made substantial progress in the understanding of mental disorders and in integrating multiple approaches into psychiatric therapy. Currently, we have specific treatments available to ameliorate affective disorders, anxiety disorders, psychoses, and substance use disorders. Despite this encouraging development, a substantial proportion of patients still fail to achieve remission and still continue to suffer from symptoms. Others may experience a poor balance between effective treatment and side effects.

Non-invasive brain stimulation techniques such as electroconvulsive therapy (ECT) have been part of the psychiatry tool kit for decades. In the past two decades, novel non-invasive methods have entered the clinical routine. For example, the use of repetitive transcranial magnetic stimulation (rTMS) or direct current stimulation (tDCS) have become widely available $[1,2]$. Even though ECT is still more commonly used than rTMS, numbers of the novel applications are increasing.

The use of novel brain stimulation techniques bears several promises to the field of psychiatry. First, it can be administered as monotherapy or as an augmentation of ongoing medication or psychotherapy. Thus, we can treat patients who are reluctant to use classic psychiatric therapy options and at the same time offer a number of additional treatment choices to patients. This is a major improvement and extension of the repertoire of psychiatrists. Examples are the use of tDCS to improve decision making in gambling

Sebastian Walther

sebastian.walther@upd.unibe.ch

1 Translational Research Center, University Hospital of Psychiatry and Psychotherapy, University of Bern, Bern, Switzerland

2 Department of Head and Skin (UZGent), Ghent Experimental Psychiatry (GHEP) Lab, Ghent University, Ghent, Belgium

3 Department of Psychiatry (UZBrussel), Free University Brussels, Brussel, Belgium

4 Department of Electrical Engineering, Eindhoven University of Technology, Eindhoven, The Netherlands disorders or for acute depression $[3,4]$, the use of doubleddosed rTMS treatment to alleviate obsessive compulsive disorder (OCD) symptoms [5]. Second, brain stimulation allows for novel combinations of treatments. Researchers are currently exploring the opportunities to use rTMS and tDCS before or during psychotherapy sessions in depression, OCD or posttraumatic stress disorder (PTSD) [6]. This mode of action extends simple augmentation of one treatment by a second one; brain stimulation might be used to specifically accelerate training effects in psychotherapy. Finally, the development of brain stimulation techniques urges scientists to focus on the pathobiology of mental disorders. These novel treatments are grounded in knowledge on the pathophysiology of symptoms such as auditory verbal hallucinations, tinnitus, pain, or psychomotor slowing [7]. Further developments will require the test of brain stimulation effects on neurophysiology, the optimal dosing, protocol design, and perhaps the identification of the ideal candidate patients for brain stimulation [8], also for FDA approved rTMS applications for depression [9]. Thus, neuroscience efforts are brought back into the clinical practice. In sum, brain stimulation offers multiple benefits to psychiatry pushing the boundaries of psychiatric therapy. Adding novel treatments, improving current psychotherapy, and focusing on problematic behaviors instead of diagnostic categories, will lead to persistent changes in the field.

Currently, we are experiencing an exciting parallel stream of exploration and confirmation, when many groups test new protocols or indications, while others already run confirmatory randomized controlled trials to accelerate implementation of the novel techniques into clinical routine. Examples are the use of transcranial magnetic stimulation in the treatment of auditory hallucinations or depression [10]. This special issue is devoted to showcase the use of brain stimulation in psychiatry. We have aggregated articles on the use of ECT, rTMS, tDCS, and transcranial alternating current stimulation (tACS). Topics cover eating disorders, depression, obsessive compulsive disorder, PTSD, schizophrenia and the effects of brain stimulation on cerebral metabolism or connectivity. We hope that readers will enjoy the selection of 
articles on non-invasive brain stimulation techniques, keeping in mind that this issue contains no report on promising invasive methods, such as vagus nerve stimulation and deep brain stimulation. In sum, this special issue will introduce some of the current discussions on brain stimulation, which is likely to be the next frontier in psychiatry.

\section{References}

1. Lefaucheur JP, Antal A, Ayache SS, Benninger DH, Brunelin J, Cogiamanian $\mathrm{F}$ et al (2017) Evidence-based guidelines on the therapeutic use of transcranial direct current stimulation (tDCS). ClinNeurophysiol 128(1):56-92

2. Rossi S, Antal A, Bestmann S, Bikson M, Brewer C, Brockmoller $\mathrm{J}$ et al (2020) Safety and recommendations for TMS use in healthy subjects and patient populations, with updates on training, ethi$\mathrm{cal}$ and regulatory issues: expert guidelines. ClinNeurophysiol S1388-2457(20):30514-30519

3. Brunoni AR, Moffa AH, Sampaio-Junior B, Borrione L, Moreno ML, Fernandes RA et al (2017) Trial of electrical direct-current therapy versus escitalopram for depression. N Engl J Med 376(26):2523-2533

4. Soyata AZ, Aksu S, Woods AJ, Iscen P, Sacar KT, Karamursel S (2019) Effect of transcranial direct current stimulation on decision making and cognitive flexibility in gambling disorder. Eur Arch Psychiatry ClinNeurosci 269(3):275-284

5. Tandt HLN, Van de Velde N, De Witte S, Audenaert K, Baeken C, Lemmens GMD (2020) Is twice daily LF-rTMS a viable treatment option for treatment-resistant OCD? Results from an open-label feasibility study. Eur Arch Psychiatry ClinNeurosci. https://doi. org/10.1007/s00406-020-01142-x

6. Bajbouj M, Padberg F (2014) A perfect match: noninvasive brain stimulation and psychotherapy. Eur Arch Psychiatry ClinNeurosci 264(Suppl 1):S27-33

7. Lefebvre S, Pavlidou A, Walther S (2020) What is the potential of neurostimulation in the treatment of motor symptoms in schizophrenia? Expert Rev Neurother 20(7):697-706

8. Kaczmarczyk M, Regen F, Heuser I, Bajbouj M, Hellmann-Regen J (2020) Inhibition of monoamine oxidase activity by repetitive transcranial magnetic stimulation: implications for inter-train interval and frequency. Eur Arch Psychiatry ClinNeurosci 270(1):119-126

9. Baeken C, Brem AK, Arns M, Brunoni AR, Filipcic I, GanhoAvila A et al (2019) Repetitive transcranial magnetic stimulation treatment for depressive disorders: current knowledge and future directions. CurrOpin Psychiatry 32(5):409-415

10. Plewnia C, Brendel B, Schwippel T, Martus P, Cordes J, Hasan A et al (2018) Treatment of auditory hallucinations with bilateral theta burst stimulation (cTBS): protocol of a randomized, doubleblind, placebo-controlled, multicenter trial. Eur Arch Psychiatry ClinNeurosci 268(7):663-673 